Association for Information Systems AIS Electronic Library (AISeL)

Wirtschaftsinformatik Proceedings 2001

Wirtschaftsinformatik

September 2001

\title{
Application Service Providing - Erfahrungsbericht aus Sicht eines Providers
}

\author{
Kai Riemer \\ Universität Münster, kai.riemer@sydney.edu.au \\ Frederik Ahlemann \\ Cataligent $\mathrm{GmbH}$, frederik.ahlemann@cataligent.com
}

Follow this and additional works at: http://aisel.aisnet.org/wi2001

\section{Recommended Citation}

Riemer, Kai and Ahlemann, Frederik, "Application Service Providing - Erfahrungsbericht aus Sicht eines Providers" (2001). Wirtschaftsinformatik Proceedings 2001. 53.

http://aisel.aisnet.org/wi2001/53 
In: Buhl, Hans Ulrich, u.a. (Hg.) 2001. Information Age Economy; 5. Internationale Tagung Wirtschaftsinformatik 2001. Heidelberg: Physica-Verlag

ISBN: 3-7908-1427-X

(C) Physica-Verlag Heidelberg 2001 


\title{
Application Service Providing - Erfahrungs- bericht aus Sicht eines Providers
}

\author{
Kai Riemer \\ Universität Münster \\ Frederik Ahlemann \\ Cataligent $\mathrm{GmbH}$
}

Zusammenfassung: Dem Application Service Providing (ASP) Konzept wird von Marktforschungsseite ein erhebliches Wachstum vorausgesagt. Entsprechend groß ist die Aufmerksamkeit, die ihm zuteil wird. Unklar bleibt jedoch oft, was ASP wirklich ist und welche Erfolgsfaktoren für das neue Konzept gelten. Der Beitrag erläutert anhand eines Erfahrungsberichtes die Konfiguration eines ASP-Leistungsangebots, die ASP-Wertkette, sowie wesentliche Erfolgsfaktoren. Der Beitrag schildert die Erfahrungen beim Aufbau der Application Services der Cataligent Inc., eines ASP im Bereich Collaboration, Projekt- und Programm-Management (www.cataligent.com). Dem vorangestellt ist eine Begriffsdiskussion sowie die Klassifikation des Konzepts zur Einordnung des Beispielfalls.

Schlüsselworte: Application Service Providing, ASP, Erfahrungsbericht, Projektmanagement, Application Hosting, IT-Outsourcing

\section{Einleitung}

\subsection{Aktuelle Situation des Application Service Providing}

Ähnlich wie im Internet- und E-Commerce-Sektor weichen bei der Beurteilung der Wachstums- und Marktpotenziale des ASP Wunschdenken und realistische Betrachtung in der jetzigen Phase des Marktzyklus teils erheblich voneinander ab. Dies liegt zum einen in einer fehlenden einheitlichen Begriffsbildung und zum anderen in einer übertriebenen Euphorie, mit der die verschiedenen Interessengruppen das Thema in den Medien platzieren. Zur Zeit befinden wir uns am Ende einer Hype-Phase im Übergang in eine Phase der Ernüchterung. Es ist absehbar und einsichtig, dass dieser Phase eine realistischere Betrachtung des Themas folgen wird, die den Markt in ein Stadium der Reife und des tatsächlichen Produktivitätsgewinns führen wird. Unternehmen ohne klaren Fokus und Positionierung, 
und solche, die es nicht verstehen, die Vorteile des Konzepts herauszustellen und zu adressieren, werden es schwer haben, am Markt zu bestehen. Der vorliegende Beitrag möchte zur Begriffskonsolidierung und Klassifikation beitragen und diese anhand eines Erfahrungsberichts illustrieren.

\subsection{Das Fallbeispiel Cataligent}

Das Unternehmen wurde im April 2000 als Spin-Off der Management-Beratung Arthur D. Little in San Francisco gegründet und bietet als Application Service Provider unter dem Produktnamen „Business Transformation Framework“ eine Projekt- und Programm-Managementplattform über das Internet an. Cataligent zielt mit seinen Leistungen in erster Linie auf Großkunden und Unternehmensberatungen, die in großen, verteilten, dezentralen Projekten einer umfassenden und flexiblen IT-Unterstützung bedürfen. Das Framework umfasst Werkzeuge zur Unterstützung des gesamten Projektlebenszyklus, vom Ideen-Management bis zu Projektpriorisierung, -planung und -steuerung. Mittlerweile hat Cataligent sein Produkt erfolgreich am Markt platziert und ist zudem auch in Europa präsent.

\section{Begriffsbildung}

Um zu einem genauen ASP-Begriffsverständnis zu kommen, gilt es ASP von Konzepten wie Outsourcing und Application Hosting abzugrenzen. Verfolgt man die aktuelle Diskussion zu ASP in der Literatur, so besteht Einigkeit darüber, dass es sich bei ASP um zentral erbrachte Leistungen handelt, bei der einer Gruppe von Kunden Software auf Mietbasis (also ohne Lizenzen) über Internet oder private Netze zur Verfügung gestellt wird:

- „An ASP deploys, hosts and manages access to a packaged application to multiple parties from a centrally managed facility." (www.aspindustry.com)

- „ASP ist ein Dienstleistungskonzept, bei dem vertraglich geregelt Unternehmen oder Privatpersonen der Zugriff auf eine zentral betriebene Applikation vermietet wird. Der Zugriff der Anwender erfolgt über das Internet oder private Netze." [BeLo00, S. 14]

Es muss jedoch festgestellt werden, dass in der momentanen Diskussion zwei Begriffsebenen miteinander vermischt werden: Die Art und Weise der Softwareoder Servicebereitstellung (Application Hosting versus Application Service) sowie die Frage nach dem Make-or-Buy der Leistungen (Insourcing versus Outsourcing). 


\subsection{Insourcing versus Outsourcing}

ASP wird häufig als Spezialfall des IT-Outsourcing betrachtet: ASP ist „,...eine besondere Form des Outsourcing, bei der die Nutzer über keinerlei Lizenzen an der Software mehr verfügen müssen. " [MeSe00, S.21] Es handelt sich jedoch bei Application Service Providing nicht wirklich notwendigerweise um einen Spezialfall des Outsourcing. Vielmehr geht es hierbei um zwei verschiedene Gegenstandsebenen. Outsourcing kennzeichnet die organisatorische Verlagerung eines Teils der Leistungserstellung auf einen externen Partner. Insourcing als Gegenteil bezeichnet die Aufnahme eines bisher extern realisierten Teils der Wertschöpfung in das Unternehmen (vertikale Integration). Wie organisatorische Entscheidungen solcher Art umgesetzt werden ist dabei völlig offen.

So kann Software auch unternehmensintern als Application Service zur Verfügung gestellt werden. (vgl. hierzu [PiJa00, S. 72]) Insbesondere für Unternehmen mit einem hohen Grad an Virtualisierung, also beispielsweise vielen verschiedenen Standorten, bietet sich die Nutzung des Internets für die Bereitstellung an. ASP ist also kein Teil von IT-Outsourcing, besitzt gleichwohl aber eine große Schnittmenge, die auch im Weiteren betrachtet werden wird.

\subsection{ASP versus Application Hosting}

Wie oben bereits angesprochen, soll ASP vom reinen Hosting und von Business Process Outsourcing abgegrenzt werden. Dies geschieht anhand der ASP-Charakteristika (vgl. [Mizo99, S. 2f.]):

- Applikationszentriert: Im Gegensatz zum Business Process Outsourcing, bei dem ein gesamter Geschäftsprozess an einen Dienstleister übertragen wird (z.B. im Personalwesen, Rechnungswesen) stehen bei ASP von zentraler Stelle bereitgestellte Software Services im Mittelpunkt. Ähnlich grenzt sich ASP von reinen Hosting Services ab. Letztere fokussieren auf Betrieb und Bereitstellung einer Hardware-/Netzinfrastruktur. Das Leistungsangebot umfasst nicht die Software selbst, sondern deren Speicherung und Bereitstellung. ASPs greifen ihrerseits häufig auf Hosting-Leistungen zurück, ohne diese selbst aufzubauen. $\mathrm{Zu}$ den Rollen vergleiche auch [TaGü00, S. 969f.]).

- Verkauf des Zugangs: Teil des ASP-Wertangebots ist, dem Kunden für bestimmte (oft unbefristete) Zeit Zugang zu den Software-Services zu gewähren. Dafür fallen i.d.R. zeit- und nutzungsabhängige Gebühren an, die jedoch keine Investitionen darstellen (,Software-as-a-Service“). Dieser Service wird grundsätzlich in Anspruch genommen bevor bzw. ohne dass eine eigene Lösung entwickelt wird (man spricht hier auch von Pre-Implementation Outsourcing). [Prin00, S. 3] Auch hier lässt sich das Application Service Providing wieder vom Application Hosting abgrenzen, bei dem ein Unternehmen bereits in die Lizensierung bzw. Entwicklung einer Software investiert hat (,Software-as-a- 
Product") und nun den Betrieb dieser Software einem Dienstleister überträgt (Post-Implementation Outsourcing).

- One-to-Many-Solution: Ein Application Service Provider bietet immer einen standardisierten (one-to-all) wenn auch oft konfigurierbaren Service (one-tomany) an, der in gleicher Form für eine Vielzahl von Kunden geeignet ist (im Sinne einer Serienfertigung). Demgegenüber handelt es sich beim klassischen IT-Outsourcing um One-to-One-Solutions, weil hier Individualleistungen erbracht werden, die in ihrer Form nur für jeweils ein Unternehmen geeignet sind (im Sinne einer Einzelfertigung). (vgl. [Knol00, S. 443])

- Externes Management: Ein Application Service wird grundsätzlich extern gemanagt, entweder von einer zentralen oder aber verschiedenen dezentralen Stellen aus, so dass der Kunde ,remote“ - meist über das Internet - auf den bereitgestellten Application Service zugreift. Niemals jedoch unterliegt die Applikation der Kontrolle des Kunden, noch wird sie vom Kunden entwickelt oder für diesen maßgeschneidert (siehe oben).

- Netzspezifische Entwicklung: Im Vergleich zum Hosting, also dem zentralen Betrieb einer klassischen Software-Lösung liegt ein wesentlicher Vorteil reiner ASP-Lösungen in der Nutzung der Spezifika des Internets. Es ergeben sich dadurch gänzlich neue Services, die einen Nutzen bieten, den klassische Software (z.B. externes Hosting von ERP- oder Office-Software) nicht bieten kann. Durch die globale, ortsunabhängige Verfügbarkeit der Services kann beispielsweise die Verteilung von Leistung und die Virtualisierung von Unternehmen unterstützt werden.

\subsection{Die ASP-Charakteristika am Praxisbeispiel}

Auf das Fallbeispiel Cataligent treffen alle fünf der genannten Merkmale zu: Cataligent fokussiert mit seinem „Business Transformation Framework“ die Bereitstellung einer Applikation und gewährt den Nutzern einen Zugang auf Mietbasis. Das Zahlungsmodell ist „Pay per User per Month“, d.h. die Mietgebühr wird für jeden Benutzer, der im Monat von den ASP Gebrauch gemacht hat, fällig. Dabei richtet sich die Gebühr nach der Benutzerrolle: Projektmanager, die eine erweiterte Funktionalität nutzen, zahlen eine höhere Gebühr als Team-Mitglieder.

Cataligent spricht mit seinen Application Services eine breite Kundengruppe an (One-to-many). Unterschiedlichen Kundenbedürfnissen wird man über die weitgehende Konfigurierbarkeit der Software gerecht, die von Benutzerverwaltung, Designgestaltung bis zur Anpassung an kundenspezifische Geschäftsprozesse reicht. Für die Nutzung wird auf Kundenseite lediglich ein Internet-Zugang und ein geeigneter Browser samt notwendigem Plug-in benötigt, da die Application Services vollständig auf bekannten Standards (HTML, Java, JavaScript) basieren. 
Diese Servicemerkmale ermöglichen dem Nutzer eine team- und projektzentrierte Arbeitsweise in verteilten Projektlandschaften, die ohne das Internet so nicht denkbar wäre. Durch die weltweite Verfügbarkeit können Projektteilnehmer unabhängig von Ort und Zeit Projekte dezentral planen, steuern, Arbeitsergebnisse teilen und miteinander kommunizieren.

\section{Das ASP-Wertangebot}

Nachdem die wesentlichen Charakteristika des ASP-Konzeptes vorgestellt und am Praxisbeispiel illustriert wurden, werden nun die spezifischen ASP-Wertangebote für den Kunden überblicksartig erläutert: Ein wesentlicher Vorteil von ASP ist die hohe Geschwindigkeit bei der Einführung der Lösung, die zu extrem kurzen Zeitabständen zwischen Bedarfsidentifizierung und Einsatz führt, da die Lösung nicht selbst entwickelt, sondern nur der passende ASP gefunden werden muss. Diese kurzen Time-to-Market-Zyklen unterstützen sowohl den notwendigen Integrationsprozess bei Fusionen als auch eine eventuelle Neuausrichtung von Unternehmen. Einfache ASP-Kostenmodelle, sowie Kostentransparenz erleichtern die Kalkulation des Einsatzes; es sind meist keine Investitionen notwendig, zudem sind die „Total Cost of Ownership (TCO)“ sehr gering (siehe Tabelle 1).

Darüber hinaus sind ASP-Lösungen aus Kundensicht sehr gut skalierbar, da für eine Ausweitung der Nutzung lediglich die Anzahl der Zugänge oder der Transaktionen erhöht wird, jedoch keine Hardwareerweiterungen notwendig werden (außer den meist ohnehin benötigten Büroarbeitsplätzen mit Netzzugang). Dies bietet die Möglichkeit, dynamisches Wachstum zu begleiten und macht ASP auch für kleine und schnellwachsende Unternehmen interessant. Hinzu kommt, dass kein eigenes IT-Know-How und -Personal aufgebaut werden muss und somit Kapazitäten und Finanzmittel für die Kernbereiche des Unternehmens gespart und die innerbetriebliche Komplexität reduziert werden können.

Ein weiterer wesentlicher Vorteil liegt in der automatischen und transparenten Einspielung von Updates, was eine jederzeit aktuelle Software garantiert. Die Konzentration auf Kernkompetenzen wird also durch ASP noch weitergehend gefördert als dies mit klassischem Outsourcing bereits der Fall ist, da bei der Nutzung von ASP ja auch die (Weiter-)Entwicklung der Software wegfällt.

Cataligent-Kunden profitieren insbesondere von einer kürzeren Time-To-MarketZeitspanne, da ein Zugang zu den Application Services innerhalb weniger Minuten verfügbar gemacht werden kann. Das Argument der geringen Total Cost of Ownership eines Application Services bedarf oft einer ausführlichen Argumentation, da es viele Kunden gewohnt sind, den „Kaufpreis“ einer Software zum Kostenvergleich heranzuziehen. Bei einem derartigen Vergleich schneiden ASPs wie Cataligent nicht in jedem Fall besser ab als Non-ASP-Konkurrenten. Nimmt man 
jedoch Kosten für Server-Hardware und -Software, Wartung, Weiterentwicklung, usw. hinzu, sind Application Services i.d.R. auch bzgl. der Kosten konkurrenzfähig.

Es bleibt anzumerken, dass Nutzern eines Application Services nahezu ausschließlich variable Kosten entstehen. Diese können bei Cataligent in der Regel direkt durch das Projektbudget gedeckt werden, so dass langwierige Genehmigungen von Investitionen zugunsten flexibler Entscheidungen entfallen können.

\begin{tabular}{|c|c|}
\hline ASP-Wertangebot & \\
\hline $\begin{array}{l}\text { Time-to-Market, Ge- } \\
\text { schwindigkeit }\end{array}$ & $\begin{array}{ll}\text { - } & \text { "Plug-and-Play“-Lösung, } \\
\text { - } & \text { kein oder sehr geringer Installationsaufwand } \\
\text { - } & \text { wenig oder kein Customizing } \\
\text { - } \quad \text { in jedem Fall: keine Entwicklung }\end{array}$ \\
\hline $\begin{array}{l}\text { Einfaches Kostenmo- } \\
\text { dell, Kostentransparenz }\end{array}$ & $\begin{array}{ll}- & \text { Keine Investitionen erforderlich } \\
\text { - } & \text { Zugriffsgebühren decken Großteil der Totalkosten und } \\
\text { sind als variabel anzusehen. }\end{array}$ \\
\hline $\begin{array}{l}\text { Geringe Kosten (TCO) } \\
\text { (Insgesamt sind die } \\
\text { TCO meist geringer als } \\
\text { bei einer Individual- } \\
\text { oder Inhouse-Lösung) }\end{array}$ & $\begin{array}{l}\text { Eingeschlossen sind i.d.R.: } \\
\text { - } \quad \text { Server-Hardware und -Software: Betriebssysteme, } \\
\text { Middleware, Datenbanken, die Applikation selbst } \\
\text { - } \quad \text { Datensicherung } \\
\text { - } \quad \text { Administration, Wartung und Updates } \\
\text { - } \quad \text { Customer-Support } \\
\text { In der Regel nicht enthalten sind: Training und Customizing }\end{array}$ \\
\hline $\begin{array}{l}\text { Dynamisches Wachs- } \\
\text { tum }\end{array}$ & $\begin{array}{l}\text { Application Services sind für den Kunden beliebig zu skalie- } \\
\text { ren, ohne dass zusätzliche Ressourcen hierzu notwendig } \\
\text { wären. }\end{array}$ \\
\hline $\begin{array}{l}\text { Mangel an qualifizier- } \\
\text { tem IT-Personal }\end{array}$ & $\begin{array}{l}\text { Für die Nutzung eines ASP ist kaum IT-Know-How nötig. Im } \\
\text { Prinzip „,teilen“ sich die Kunden eines ASP dessen Experten. }\end{array}$ \\
\hline Komplexitätsreduktion & $\begin{array}{l}\text { Reduktion der unternehmensinternen Komplexität und Kon- } \\
\text { zentration auf Kernkompetenzen }\end{array}$ \\
\hline
\end{tabular}

Tabelle 1: ASP-Wertangebote

\section{Die ASP-Wertschöpfungskette}

Nach dem der Begriff des ASP abgegrenzt und das ASP-Wertangebot aufgezeigt wurde, wird das ASP-Konzept im weiteren hinsichtlich der Umsetzung und verschiedener Ausprägungen näher beleuchtet. Zunächst wird die ASP-Wertschöpfungskette vorgestellt, dabei werden die Rollen und Aufgaben exemplarisch am Beispiel von Cataligent erläutert (siehe auch Abbildung 1). 


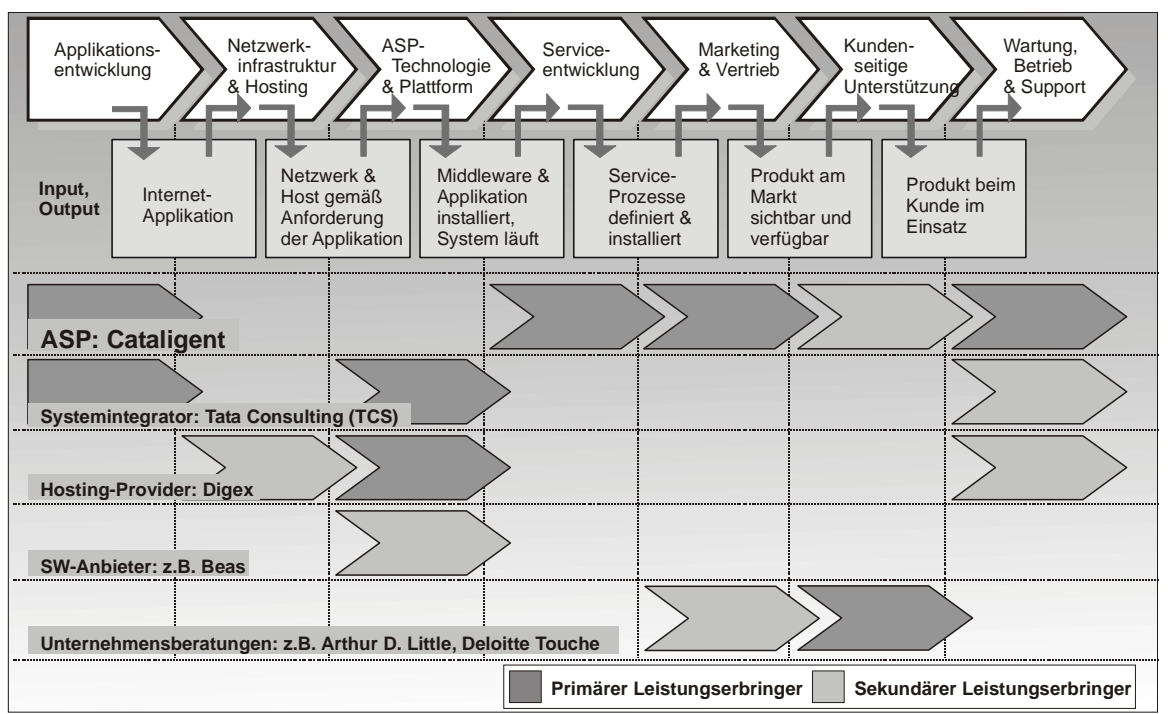

Abbildung 1: Die ASP-Wertkette, mit spezifischer Rollenverteilung im Fall von Cataligent (angelehnt an [PiBu00, S. 47]).

\subsection{Entwicklung und Architektur}

Grundlage jedes Application Services ist die Software-Applikation, deren Spezifikation und Entwicklung eine der ersten Aufgaben eines neuen ASP darstellt. Diese Aufgabe wurde bei Cataligent dadurch erleichtert, dass ein umfassender Prototyp bei der Gründung des Unternehmens verfügbar war. Dieser konnte neben fachlich-methodischen Grundlagen von der Ursprungsfirma Arthur D. Little übernommen werden, was hat nicht allein die Spezifikationsphase verkürzt, sondern zudem das Risiko des Scheiterns verringert hat. Die eigentliche Entwicklung der Applikation wurde in Kooperation mit dem indischen Partner Tata Consultancy Services (TCO) durchgeführt, wobei kurze Kommunikationswege und ein strenges Controlling der Entwicklung durch permanente Präsenz indischer Experten in San Francisco und regelmäßige Besuche von Cataligent-Mitarbeitern in Indien sichergestellt wurden.

Die zweite große Leistungskomponente eines ASP besteht in der Bereitstellung von Netzinfrastruktur und Hosting-Umgebung. Da Cataligents Kernkompetenzen fachlich-methodischer Natur sind, wird diese Leistungskomponente durch den externen Application-Hosting-Provider Digex übernommen. Bei der Selektion des Partners wurde insbesondere auf internationale Präsenz Wert gelegt, um in einer späteren Phase die Application Services nicht nur von zentraler Stelle in den USA, sondern auch wegen der besseren Geschwindigkeit und Verfügbarkeit, direkt innerhalb der weiteren Ziel-Lokationen anbieten zu können. 
Der technische Aufbau des Application Service ist eine Aufgabe an der eine Vielzahl von Partnern mitarbeiten. Da hier die Hardware-Plattform inkl. Betriebssystem, die Middleware und die eigentliche Applikation integriert werden, für die jeweils ein anderer Lieferant verantwortlich ist, ist hier besondere Aufmerksamkeit des Service Providers gefragt. Besonders im Hinblick auf garantierte Betriebszeiten ist ein nicht unerheblicher Konfigurations- und Testaufwand einzuplanen. Im Cataligent-Fall wurden für die komplette Installation und den Test ca. vier Wochen veranschlagt, nach denen das System auch genutzt werden konnte. Der eigentliche Abschluss der Arbeiten hat jedoch vier weitere Wochen in Anspruch genommen.

Die Cataligent Architektur ist als klassische dreischichtige Client-Server-Architektur realisiert. Sowohl der Datenbank- als auch der Applikations-Server laufen unter einem Solaris-System, der Web-Server unter Windows NT. Sämtliche Systemkomponenten sind physisch redundant ausgelegt, so dass beim Ausfall einer Komponente der Betrieb weiterhin sichergestellt ist. Zudem sorgt ein sog. LoadBalancer für eine Verteilung der Benutzeranfragen auf jeweils diejenige Komponente mit der aktuell geringsten Auslastung.

\subsection{Service, Beratung und Vertrieb}

Neben den Application Services müssen entsprechende Serviceprozesse geplant und realisiert werden. Diese umfassen die Bereitstellung einer Service-Hotline, sowie die prozessuale Unterstützung des gesamten Kundenprozesses, von der Akquisition bis hin zur Beendigung des Vertragsverhältnisses. Cataligent legt auf diesen Bereich viel Wert, um langfristige Beziehungen zu Endkunden und Vertriebspartnern aufzubauen, da die Switching-Cost bei ASP nicht so hoch sind wie bei anderen Softwarelösungen (z.B. ERP-Systemen), somit also von einer stabilen Kundenbeziehung nicht schon aufgrund natürlicher Lock-In-Effekte ausgegangen werden kann.

Im Gegensatz zu klassischen Softwareherstellern oder anderen ASPs realisiert Cataligent ein indirektes Marketing- und Vertriebssystem. Die Application Services werden von Unternehmensberatungen als ergänzender Service in Beratungsprojekten oder sogar als eigenständiges Beratungsprodukt angeboten. Auf diese Weise kann Cataligent einen großen Teil des Beratungs- und Konfigurationsaufwandes an externe Partner abgeben und die Abhängigkeit von großen Vertriebseinheiten verringern.

Bei der laufenden Kundenbetreuung und Betrieb und Wartung des Systems nimmt Cataligent - ähnlich wie beim Aufbau - eine stark koordinierende Funktion ein. Da auftretende Probleme bei jedem der Leistungserbringer der ASP-Wertkette ihren Ursprung haben können, müssen Problemquellen schnell identifiziert und an den entsprechenden Partner weitergeleitet, sowie Antworten bzw. Lösungen an den Kunden kommuniziert werden. 


\section{Typen und Klassifikation von Application Services}

Nachdem im letzten Kapitel die typische ASP-Wertkette vorgestellt wurde, werden nun verschiedene Arten von Application Service Providern vorgestellt. ASP lassen sich dabei anhand verschiedener Kriterien klassifizieren. Die folgende Klassifikation soll einen Überblick über am Markt zu beobachtende Strömungen geben und zur Einordnung des Leistungsangebotes von Cataligent dienen. Der morphologische Kasten am Ende dieses Kapitels gibt einen abschließenden Überblick über mögliche Ausdifferenzierungen des ASP-Konzepts (siehe hierzu Tabelle 2).

\subsection{Klassifikation anhand des Spezialisierungsgrades}

Eine gängige Klassifikation unterteilt ASP-Leistungen nach den Branchen und betrieblichen Funktionsbereichen für die die Lösungen bereitgestellt werden. Die Funktionsbereiche können dabei z.B. nach der klassischen Wertkette von Porter gegliedert werden. Dueck führt als dritte Dimension noch die Größe der jeweils adressierten Unternehmen an [Duec00, S. 4]. Es kann zum einen Spezialanbieter geben, die entweder Funktions- oder Branchenspezialisten sind, oder aber sich auf eine Nische (z.B. Beschaffung im Chemiebereich) beschränken. Zum anderen gibt es Universalanbieter, die Basistechnologien bereitstellen, die unabhängig von betrieblichen Funktionsbereichen sind, wie Kommunikation und Conferencinglösungen (z.B. Hotmail, WebEx), Office-Software-Services (Sun, Microsoft) oder eben die Projektmanagementlösung von Cataligent. Eine weitere Gruppe von Universalanbietern bietet lediglich ein hinsichtlich Branche und Funktion undifferenziertes Portfolio von Lösungen an.

Cataligent nimmt gemäß dieser Klassifikation zwei Rollen ein und kann sowohl als Universalanbieter wie auch als Spezialanbieter gesehen werden: Die generische Projektmanagementplattform bietet Funktionen, die unabhängig von einer Branche oder einer betrieblichen Funktion sind, wie z.B. Projektplanung (Struktur-, Zeit-, Ressourcen, Kosten- und Nutzenplanung), Projektsteuerung (StatusReporting, Entscheidungsmanagement) und Kollaboration (Diskussionsforen, Dokumentenaustausch, Conferencing). Darüber hinaus nimmt Cataligent die Rolle des Spezialanbieters ein, wenn Werkzeuge angeboten werden, die spezielle branchen- und/oder funktionsspezifische Projekttypen unterstützen; dies geschieht z.B. für Softwareentwicklung (Release- und Requirements-Management), Beschaffung (Strukturierte Lieferantenauswahl) oder den Bereich Finanzen (Business Planning). 


\subsection{Klassifikation anhand des Standardisierungsgrades}

ASP Lösungen weisen, wie oben bereits angesprochen, von Natur aus einen gewissen Standardisierungsgrad auf, der die Realisierung von Skaleneffekten ermöglicht. Der ASP-Anbieter sieht sich dabei jedoch dem Trade-off aus kostengünstiger Massenfertigung und der bestmöglichen Befriedigung von Kundenwünschen gegenüber. Aus diesem Grund kann ein leistungsfähiges und einfach konfigurierbares Customizing einen wesentlichen Erfolgsfaktor darstellen. ASP-Lösungen können also nach dem Grad ihres Standardisierungsgrades und ihrer Konfigurierbarkeit klassifiziert werden. Dabei kann ein Kontinuum aufgespannt werden, das von weitgehend standardisierten Massendiensten (z.B. Hotmail) im Sinne des „one-to-all“ bis zu spezifische customizebaren Lösungen (z.B. Cataligent) im Sinne des „one-to-many“-Gedankens reicht. Zur Erinnerung: Eine „one-to-one“Lösung kann nicht als ASP bezeichnet, sehr wohl aber per Application Hosting angeboten werden.

Cataligent bietet einen Application Service, der besonders weitgehend an Kundenbedürfnisse angepasst werden kann. Ein großer Vorteil ist, dass eine Konfiguration des Systems zwar machbar, aber nicht unbedingt notwendig ist, da Standard-Einstellungen eine sofortige Nutzung des Services wie auch eine spätere Modifikation der Konfiguration ohne Probleme ermöglichen. Da unterschiedliche Projekte oft auch unterschiedlicher Anpassungen der Projektmanagementlösung bedürfen, lässt sich die Applikation zudem projektspezifisch, d.h. nicht nur auf Ebene des Cataligent-Kunden anpassen.

\subsection{Klassifikation anhand der Komplexität und des Umfangs der Services}

ASP-Angebote lassen sich außerdem hinsichtlich ihres Leistungsumfanges klassifizieren. Hier kann ein ASP-Performance-System aufgebaut werden, das von Kernleistungen bis zu kompletten Angeboten inklusive Beratung reichen kann [BeBi91]. In Anlehnung an [Mizo99] kann hier ein dreistufiges Leistungssystem aufgebaut werden (siehe Abbildung 2):

1. Core Services (einfacher Basisservice): Die ASP-Kernleistungen umfassen das Hosting der Applikation, das Monitoring und die Wartung. Der Anbieter garantiert die ständige Verfügbarkeit, entwickelt die Services weiter und spielt Updates und Upgrades ein. Darüber hinaus gehört meist ein Basissupport der Anwender zu den Leistungen. Damit sind alle ASP konstituierenden Merkmale vorhanden.

2. Managed Service: Neben den Basisservices übernimmt der ASP weitere Leistungen wie spezielle (auch kundenbezogene) Skalierung der Services hinsichtlich Geschwindigkeit, weitgehenden technischen Support, sowie die Sicherung der kundenindividuellen Daten. Im wesentlichen stehen hier Leistun- 
gen im Vordergrund, die für einen stabilen, gleichbleibend schnellen und sicheren betrieblichen Einsatz notwendig sind. Diese Leistungen werden den Kunden dann vom ASP in speziellen Service Level Agreements (SLA) zugesichert.

3. Extended Services: Die Extended Services erweitern das Leistungsspektrum um die oben angesprochenen Konfigurations- bzw. Customizing-Möglichkeiten, sowie um Training, Coaching und Ausbildung der Anwender. Weitergehende Konzepte umfassen zudem Professional Services wie Strategie und Planung des Einsatzes der ASPs, sowie weitergehende Prozessberatung.

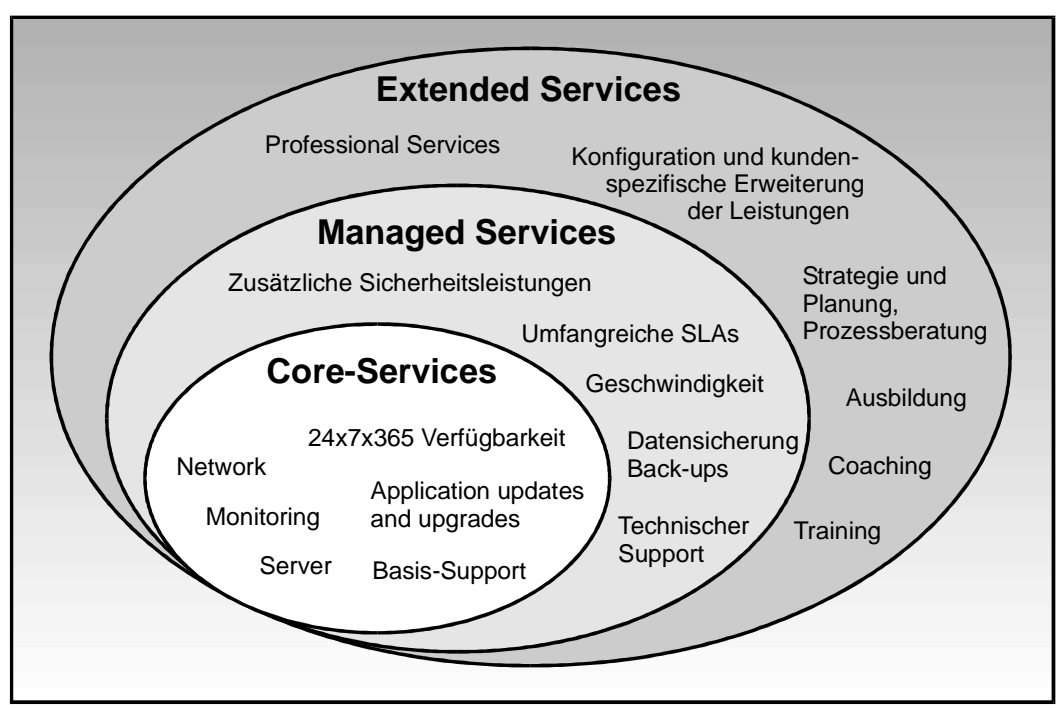

Abbildung 2: Das ASP-Leistungssystem (angelehnt an [Mizo99, S. 5f.]).

Cataligent bietet einen Extended Service an, da sich das Leistungsspektrum nicht allein in der einfachen Bereitstellung des Zugangs erschöpft, sondern die Verfügbarkeit des Service in SLAs vertraglich geregelt wird. Zudem bietet Cataligent (insbesondere über die Distributionspartner) ergänzende Prozess- und Organisationsberatung zu den Themen Projekt- und Programm-Management.

\subsection{Klassifikation anhand des notwendigen Beratungsaufwandes}

Es gibt ASP-Lösungen, deren Einsatz ohne eine vorher erfolgte Beratung nicht unmittelbar nutzenstiftend ist. Oft ist zusätzliche Prozessberatung für einen optimalen Einsatz notwendig oder sinnvoll, die über das Customizing oder simple Anwendertrainings hinaus geht. Auch hier kann ein Kontinuum aufgespannt werden, das jedoch quer zu dem oben angeführten Kontinuum von Standardisierung 
und Konfigurierbarkeit liegt. Auch standardisierte Lösungen können komplex sein und Beratungsaufwand erfordern.

Der Beratungsaufwand reicht dabei von simplen Anwendertrainings im Umgang mit der Lösung über Customizing-Beratung bis hin zur Prozess- und Organisationsberatung. Hier nimmt die ASP-Lösung beispielsweise eine Unterstützungsfunktion ein und bildet den Kern eines Prozesses, dessen Verständnis jedoch darüber hinaus weiteres Know-How erfordert. Damit kann der Übergang von der Nutzung einer ASP-Lösung hin zum Business Service Providing (BSP) gegeben sein, bei dem nicht mehr die Software-Services im Zentrum der Dienstleistung stehen, sondern vielmehr ein gesamter Geschäftsprozess. Zur Erbringung der Leistung ist dann weit mehr notwendig als die Bereitstellung eines Application Services. [Mizo99, S. 2] In der Tat prognostizieren einige Analysten, dass ASP nur ein Zwischenschritt beim Übergang zum Business Service Providing ist, bei dem nicht Software sondern Prozesse vom Provider aus der Distanz in möglichst standardisierter und in Software implementierter Form angeboten werden. [Prin00, S. 1]

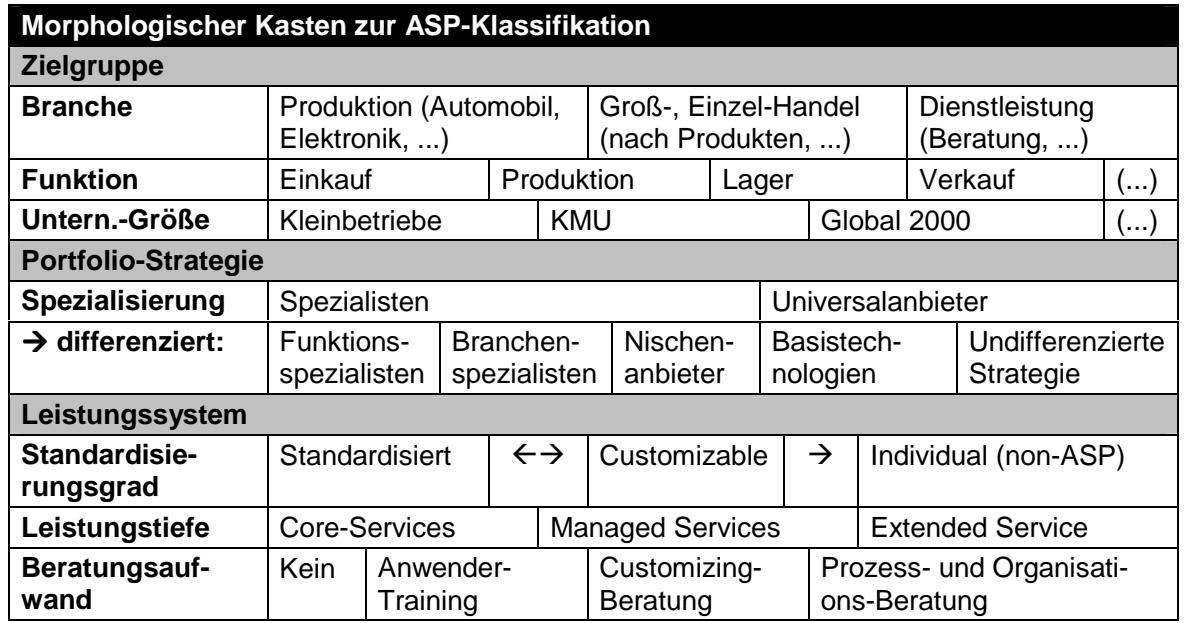

Tabelle 2: Morphologischer Kasten zur Klassifikation von ASP

Der Beratungsaufwand bei der Nutzung der Cataligent Application Services schwankt stark und ist abhängig von der Projektmanagement-Kompetenz des Kunden. Die Erkenntnis, dass ein schlechter Projektmanager durch Werkzeugunterstützung nicht zum guten Projektmanager wird, hat dazu geführt, dass das Leistungsspektrum von Cataligent nicht nur den Application Service sondern auch Organisations- und Prozessberatung zum allgemeinen Projektmanagement umfasst. Darüber hinaus vermittelt Cataligent spezielles Prozesswissen beim Einsatz der erweiterten branchen- bzw. funktionabhängigen Werkzeuge. In diesen Bereichen profitiert Cataligent von seinen Kernkompetenzen und seiner engen Zusammenarbeit mit Beratungsunternehmen. 


\section{Erfolgsfaktoren}

Zusammenfassend kann man sagen, dass zur Erstellung eines guten Application Service einerseits die bereits angesprochenen Wertangebote für den Kunden möglichst gut eingelöst werden müssen und andererseits die Nachteile und Hindernisse des Konzepts gegenüber dem Kunden offen angesprochen und weitgehend abgefedert werden müssen.

Besondere Bedeutung kommt hier der Gestaltung der Service Level Agreements (SLA) zu, um eventuelle Ängste des Kunden zu reduzieren, die sich aus der Distanz zum Anbieter und der Nutzung öffentlicher Netze (Internet) ergeben. Kernfaktoren liegen hier in den für den Kunden sensiblen Bereichen wie Sicherheit (Übertragung und Datensicherung), Privacy und Datenschutz

Fast ebenso wichtig sind die vom Kunden möglicherweise wahrgenommenen Lock-In-Effekte (Angst vor Wechselkosten) bei der Bindung an einen ASP. Hier müssen z.B. Exportformate für die Daten bereitgestellt und Möglichkeiten zur Portierung auf andere Lösungen bei eventueller Vertragsbeendigung aufgezeigt werden. Cataligent bietet daher seinen Kunden einen kompletten Datenexport in Dateien nach jeweiligem Industriestandard, sowie vollständige Ausdrucke.

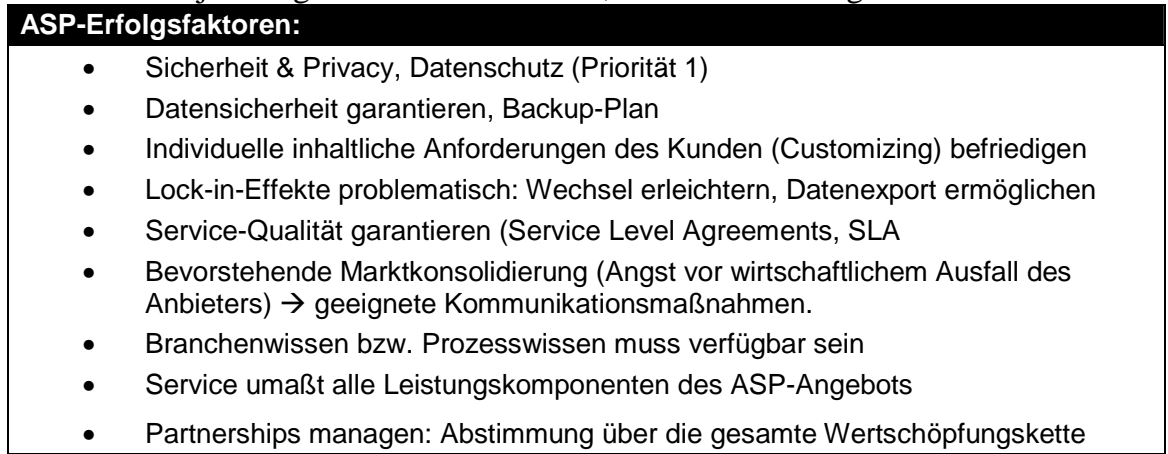

Tabelle 3: ASP-Erfolgsfaktoren

Aus der Erfahrung von Cataligent lässt sich zudem sagen, dass dem Management externer Partner beim Aufbau eines ASP eine besondere Bedeutung zukommt. Cataligent hat die Erfahrung gemacht, dass die Gestaltung des Netzwerkes und die Koordination der ASP-Wertkette deutlich aufwendiger ist als ursprünglich vermutet. Dies gilt umso mehr, da die Kunden ein integriertes Leistungspaket erwarten, das sie aus der Hand eines Lieferanten erhalten. Die Aufgaben beim Aufbau eines Application Services Providing beschränken sich also längst nicht auf die Entwicklung eines guten Konzeptes und dessen Umsetzung. Mindestens ebenso entscheidend ist die Fähigkeit, die Komponenten aller Partner für den Kunden zu einem homogenen Produkt werden zu lassen. 


\section{Zusammenfassung}

Ausgehend von der aktuellen Situation des Application Service Providing hat der Beitrag gezeigt, wie das Konzept defininiert, charakterisiert, klassifiziert und von verwandten Konzepten abgegrenzt werden kann. Dabei wurde immer beispielhaft Bezug genommen auf die Services des Fallbeispiels Cataligent. Ferner wurde anhand des Praxisbeispiels deutlich gemacht, was die wesentlichen Erfolgsfaktoren beim Aufbau eines Application Service sind und welche Aspekte notwendig sind, um das Konzept sowohl für den Anbieter als auch für den Kunden zu einem Erfolg werden zu lassen.

\section{Literatur}

[BeBi91] Belz, Christian; Bircher, Bruno; et. al. (1991): Erfolgreiche Leistungssysteme, Stuttgart: Schäffer Verlag für Wirtschaft und Steuern, 1991.

[BeLo00] von Bechtolsheim, Matthias; Loth, Bettina (2000): Chancen und Risiken für Anwender und Anbieter von Application Service Providing. In: IM - Information Management \& Consulting, 15 (2000) Sonderausg., S. 14-20.

[Duec00] Dueck, Peter (2000): How hard will ASPs bite the IT Industry?. In: Gartner Group, Proceedings of the Gartner Symposium ITxpo 2000, Cannes, 2000.

[Knol00] Knolmayer, Gerhard F. (2000): Application Service Providing (ASP). In: Wirtschaftsinformatik, 42 (2000) 5, S. 443-446.

[MeSe00] Meitner, Helmut; Seufert, Jörg Arne (2000): Anforderungen an die Markteintrittsstrategie von Application Service Providern. In: IM - Information Management \& Consulting, 15 (2000) Sonderausg., S. 21-27.

[Mizo99] Mizoras, A., et. al. (1999): Worldwide ASP Market Forecast and Analysis, 19992004, IDC.

[PiBu00] Picot, Arnold; Buttermann, Anne; Walters, Robert (2000): Erfolgsfaktoren für Application Service Providing. In: IM - Information Management \& Consulting, 15 (2000) Sonderausg., S. 45-51.

[PiJa00] Picot, Arnold; Jahn, Andreas (2000): ASP als innerbetriebliches Allheilmittel? In: IM - Information Management \& Consulting, 15 (2000) Sonderausg., S. 72-77.

[Prin00] Pring, Ben (2000): ASP opportunities. In: Gartner Group, Proceedings of the Gartner Symposium ITxpo 2000, Cannes, 2000.

[TaGü00] Tamm, Gerrit; Günther, Oliver (2000): Business Models for ASP Marketplaces. In: Hansen, Hans Robert; Bichler, Martin; Mahrer, Harald (Hrsg.), Proceedings of the 8th European Canference on Information Systems (ECIS), S. 968-975, Wien, 2000. 